\title{
Migraine and temporomadibular disorders
}

\section{Migranea e disfunção temporomandibular}

José G. Speciali

\author{
1 Universidade de São Paulo, \\ Faculdade de Medicina de Ribeirão \\ Preto, Neurologia, Ribeirão Preto \\ SP, Brasil. \\ Correspondence: \\ José G. Speciali \\ Rua dos Tucunarés, 735, \\ Quinta da Alvorada; 14110-000 \\ Ribeirão Preto SP, Brasil; \\ E-mail: jgspecia@fmrp.usp.br \\ Conflict of interest: \\ There is no conflict of interest to \\ declare. \\ Received 20 March 2018 \\ Accepted 27 March 2018.
}

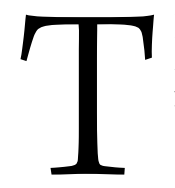

he association of temporomandibular disorders (TMD) and headache in the same patient has been found in studies and in specialized clinics, with similar results being obtained in these clinics and in the population samples ${ }^{1,2,3}$.

In a review by Speciali and $\mathrm{Dach}^{1}$, one study conducted in a specialized headache clinic, of women with migraine and chronic migraine, showed that the frequency of TMD was higher in the groups with migraine and chronic migraine than in the control group with no migraine (33.3\% for the control group against $86.8 \%$ and $91.3 \%$ for the groups with migraine and chronic migraine. The relationship was higher for muscular TMD than for the other forms of TMD. In another study of a series of 300 individuals evaluated at an orofacial pain clinic, individuals with myofascial TMD had a greater chance of having chronic daily headaches than participants without TMD, migraine, and with episodic tension-type headache, and the intensity of TMD pain was associated with a higher frequency of headaches. Thus, studies conducted at specialized clinics have demonstrated that headaches and TMD occur at a higher percentage when the two diseases are associated in the same patient compared with the respective control groups consisting of patients with only one of these diseases ${ }^{1}$.

Population studies confirm the results of studies conducted at headache and orofacial pain clinics: namely, a greater prevalence of headache in patients with TMD and vice versa. Thus, TMD and migraine/chronic migraine/chronic daily headaches/tension type headache are bidirectional comorbid diseases. Therefore, TMD aggravates primary headaches and these aggravate $\mathrm{TMD}^{1,3}$.

The possible explanation for this association is that these diseases involve the same nociceptive system. The first neurons involved in headaches are related to the first branch of the trigeminus and to the trigeminocervical complex, and those involved in TMD are related to the neurons of the first and second branches of the trigeminus. This nociceptive information converges toward the caudal nucleus of the trigeminus, and from there the pathways of headache and TMD share specific central pathways involved in pain modulation, among them the thalamus, brainstem nuclei, sensory cortex, and limbic system. Nociceptive afferents caused by headache may sensitize the brain regions related to TMD pain and facilitate the triggering or aggravation of TMD pain and vice versa ${ }^{3}$.

Effective treatments for migraine and therapies for TMD exist and have been published extensively. Nonetheless, the best approach for patients with both disorders is a point of controversy. Should one be treated first (and if so, which), or is treating both necessary?

A study by Gonçalves et al. ${ }^{2}$, in patients with migraine according to the ICHD-2, and TMD according to the Research Diagnostic Criteria for TMD - Axis I, defined the primary endpoint as a change in headache days from baseline and the secondary endpoint as a change in days with at least moderate headache from baseline. The sample consisted of 89 women with both conditions. They were randomized to receive one of four treatments. Group I: propranolol 30 $\mathrm{mg} /$ day (tid) and a stabilization splint; Group 2: propranolol and a non-occlusal (placebo) splint; Group III: placebo and a stabilization splint; Group IV: placebo and a non-occlusal splint. Evaluations were made at baseline, at the end of the blinded-phase (three months) and at the end of the open extension (six months). For reduction of headache days at three months (primary endpoint) treating both conditions (Group 1) yielded significantly better results (-5.4 days) relative to other groups (propranolol only: -3.2; occlusal splints only: -4.1 and placebo: $-3.5 ; \mathrm{p}<0.05$ ). For the secondary endpoint, differences were also significant after three months of treatment with those treating both conditions having additional benefits relative to 
all other groups. As for disability, those treating both conditions also had significant improvements relative to all other groups. These findings suggested that in patients with comorbid TMD and migraine, the best migraine outcomes happened when TMD and migraine were treated simultaneously.

In this edition of Arquivos de Neuro-Psiquiatria, Bruno and Krymchantowski ${ }^{4}$ present an interesting article: "Amitriptyline and intraoral devices for migraine prevention: a randomized comparative trial". The objectives of this study were to assess the efficacy of the Nociceptive Trigeminal Inhibition Tension Suppression System (NTI-tss) (a type of splint approved by the Food and Drug Administration for migraine prophylaxis) in reducing the frequency of migraine without aura attacks and to compare its efficacy against the use of a traditional preventive migraine drug (amitriptyline). The participants were adults, aged between 18 and 70 years, with migraine with no aura and no acute signs and symptoms of TMD. The study differs from most of those published in the literature because the patients in this study had no signs and symptoms of acute TMD. Their conclusion was that amitriptyline was significantly more effective in reducing the frequency of headache attacks of migraine without aura than the NTI-tss and the placebo non-occlusal splint. The NTI-tss showed similar results to those of the nonocclusal splint.

\section{References}

1. Speciali,JG, Dach F. Temporomandibular dysfunction and headache disorder. Headache. 2015 Feb;55 Supl 1:72-83. https://doi.org/10.1111/head.12515

2. Gonçalves DAG, Camparis CD, Franco AL, Fernandes G, Speciali JG, Bigal ME. How to investigate and treat: migraine in patients with temporomandibular disorders. Curr Pain Headache Rep. 2012 Aug;16(4):359-64. https://doi.org/10.1007/s11916-012-0268-9
3. Graff-Radford SB, Abbott JJ. Temporomandibular disorders and headache. Oral Maxillofacial Surg Clin N Am. 2016 Aug;28(3):335-49. https://doi.org/10.1016/j.coms.2016.03.004

4. Bruno, MAD \& Krymchantowski, AV. Amitriptyline and intraoral devices for migraine prevention: a randomized comparative trial. Arq Neurpsiquiatr 2018;76(4):213-18. https://doi.org/10.1590/0004-282X20180023 